\title{
Detection of a respiratory coronavirus from tissues archived during a pneumonia epizootic in free-ranging Pacific harbor seals Phoca vitulina richardsii
}

\author{
Hendrik H. Nollens ${ }^{1,2, *}$, James F. X. Wellehan ${ }^{1}$, Linda Archer ${ }^{1}$, Linda J. Lowenstine ${ }^{3}$, \\ Frances M. D. Gulland ${ }^{4}$ \\ ${ }^{1}$ Marine Mammal Health Program, College of Veterinary Medicine, University of Florida, Gainesville, Florida 32610, USA \\ ${ }^{2}$ Hubbs-SeaWorld Research Institute, San Diego, California 92109, USA \\ ${ }^{3}$ Department of Pathology, Microbiology, and Immunology, School of Veterinary Medicine, University of California, Davis, \\ California 95616, USA \\ ${ }^{4}$ The Marine Mammal Center, 1065 Fort Cronkhite, Sausalito, California 94965, USA
}

\begin{abstract}
In June 2000, 21 adult harbor seals Phoca vitulina richardsii were found dead along a localized section of the central California coast. Necropsy of 5 fresh carcasses revealed pulmonary congestion, consolidation, and hemorrhage. Histopathological changes in lungs from 2 of these seals included a necrotizing lymphocytic and histocytic lobar pneumonia with intra-lesional bacteria. A coronavirus (CoV) was detected in archived tissues from 1 of the 5 seals via a degenerate PCR for nidoviral RNA-dependent RNA polymerase (RdRp), and was subsequently confirmed via specific PCR. Based on the partial RdRp sequence, the CoV was identified as a novel, divergent member of the CoV group 1a. The virus is tentatively named harbor seal coronavirus (HSCoV). The clinical significance of $\mathrm{HSCoV}$ and its involvement in the etiology of the epizootic pneumonia and deaths of the harbor seals is uncertain.
\end{abstract}

KEY WORDS: Harbor seal $\cdot$ Phoca vitulina richardsii $\cdot$ Coronavirus $\cdot$ Pneumonia

\section{INTRODUCTION}

Since the emergence and discovery of the coronavirus $(\mathrm{CoV})$ associated with human severe acute respiratory syndrome (SARS) in 2002, CoVs have gained considerable notoriety. The SARS virus is thought to be of zoonotic origin from a wild animal reservoir (Li et al. 2005). This has led to a renewed interest in CoVs of animals, and a need for a better understanding of $\mathrm{CoV}$ transmission among species and clarification of pathogenesis of CoV infections (Saif 2004). Since 2002, a number of novel animal CoVs have been detected from both domesticated animals and wildlife (Carrington et al. 2008, Decaro \& Buonavoglia 2008, Woo et al. 2009).
CoVs are a genus of enveloped viruses with a linear positive stranded RNA genome. They are classified together with the genus Torovirus in the family Coronaviridae of the order Nidovirales. The genus Coronavirus has been divided into 3 groups (González et al. 2003). It has been proposed that Group I be subdivided into Groups 1a and 1b (Woo et al. 2007); Group 1a has only been found in laurasiatherian mammal hosts, primarily those from the Carnivora, whereas Group 1 b has been found in both laurasiatherian and euarchontoglire mammal hosts. It has been proposed that Group 2 be divided into the following groups: Group 2a, which has been found in both laurasiatherian and euarchontoglire mammal hosts; Group 2b, which contains SARS and viruses from microchiropteran bats; Group 2c, from mi- 
crochiropteran bat hosts; and Group 2d, from megachiropteran bat hosts (Woo et al. 2007). It has been proposed that Group 3 be divided as follows: Groups 3a, from avian hosts; Group 3b, which contains a coronavirus from a beluga whale Delphinapterus leucas, the only coronavirus identified in a marine mammal to date; and Group 3c, which contains viruses from diverse avian hosts as well as one from an Asian leopard cat Prionailurus bengalensis that may represent recent host switching (Woo et al. 2009).

In late May and early June 2000, 21 adult harbor seals Phoca vitulina richardsii were found dead along a localized 10 mile $(16 \mathrm{~km})$ section of the central California coast at Point Reyes National Seashore. Upon postmortem examination of 5 relatively fresh carcasses, all seals had grossly abnormal lungs, and histological examination of 3 cases revealed severe pneumonia. A similar event with similar gross necropsy findings had occurred in 1997, when approximately 90 seals were found dead in the same area, but no conclusive etiology for the event was identified. In both events, a viral infection with secondary bacterial pneumonia was suspected. CoVs are recognized causes of enteric and respiratory infections that are often fatal in young animals. Here we report on the identification of a novel respiratory coronavirus from one of these harbor seals. This novel coronavirus of wildlife is a novel member of Group 1a and is distinct from, but most closely related to the CoVs of ferrets, cats, dogs, and swine.

\section{MATERIALS AND METHODS}

Animals and samples. On 26 May 2000, a harbor seal (HS D62) was found dead at McClure's Beach, Point Reyes National Seashore, in central California, and a field necropsy was conducted. Tonsil, stomach, cerebellum, brain stem, and lymph node were collected in $10 \%$ formalin for histological analysis. An additional 4 adult harbor seals were found dead on 6 June 2000 . Two harbor seals, designated as HS 4 and 5, were fresh dead. Complete sample sets were collected in $10 \%$ formalin for histological examination, and the lung was frozen at $-80^{\circ} \mathrm{C}$. Two other seals, HS 1 and 6 , were moderately decomposed, and the lung only was collected for archiving at $-80^{\circ} \mathrm{C}$. On 8 June, a sixth fresh dead seal was found, HS 17, and tissues were collected for histology in $10 \%$ formalin, and lung archived at $-80^{\circ} \mathrm{C}$.

Degenerate and specific PCR. In 2008 RNA was extracted from the archived frozen lungs of the 5 harbor seals (HS 1, 4, 5, 6, and 17) using the RNeasy Mini Kit (Qiagen). RT-PCR for a conserved region of the coronaviral RNA-dependent RNA polymerase (RdRp) was performed using the OneStep RT-PCR Kit (Qiagen) using previously described primers $2 \mathrm{Bp}$ and $4 \mathrm{Bm}$
(Stephensen et al. 1999) on all 5 RNA extracts. The mixtures were amplified in a thermal cycler (PX2, Thermo Hybaid) with an initial reverse transcription at $40^{\circ} \mathrm{C}$ for $45 \mathrm{~min}, 95^{\circ} \mathrm{C}$ for $15 \mathrm{~min}$, followed by 5 cycles

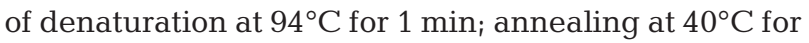
$2 \mathrm{~min}$, DNA extension at $72^{\circ} \mathrm{C}$ for $1 \mathrm{~min}$, then 40 cycles of denaturation at $94^{\circ} \mathrm{C}$ for $1 \mathrm{~min}^{\text {i annealing at }} 46^{\circ} \mathrm{C}$ for $1.5 \mathrm{~min}$, DNA extension at $72^{\circ} \mathrm{C}$ for $1 \mathrm{~min}$, with a final extension step at $72^{\circ} \mathrm{C}$ for $10 \mathrm{~min}$. The PCR products were resolved in $1 \%$ agarose gels. The bands were excised and purified using the QIAquick gel extraction kit (Qiagen). Direct sequencing was performed using the Big-Dye Terminator Kit (Perkin-Elmer) and the above second-round primers, and analyzed on ABI 3130 automated DNA sequencers at the University of Florida Interdisciplinary Center for Biotechnology Research Sequencing Facilities. All products were sequenced at least 3 times in both directions. Primer sequences were edited out prior to further analyses. Based on this edited sequence, the specific forward primer harbor seal coronavirus (HSCoV)-F1 5'-CCA AGG CTA GGG CTC GCA CT-3' and specific reverse primer HSCoV-R1 5'-CAT TAT CTA CGC CTA AAG TGA G-3' were designed. Specific RT-PCR was performed using the OneStep RT-PCR Kit (Qiagen) using the specific primers HSCoV-F1 and HSCoV-R1 on all 5 RNA extracts. The mixtures were amplified with an initial reverse transcription at $40^{\circ} \mathrm{C}$ for $45 \mathrm{~min}, 95^{\circ} \mathrm{C}$ for $15 \mathrm{~min}$, followed by 40 cycles of denaturation at $94^{\circ} \mathrm{C}$ for $1 \mathrm{~min}$; annealing at $51^{\circ} \mathrm{C}$ for $1 \mathrm{~min}$, DNA extension at $72^{\circ} \mathrm{C}$ for $1 \mathrm{~min}$, with a final extension step at $72^{\circ} \mathrm{C}$ for $10 \mathrm{~min}$. The PCR products were resolved in $1 \%$ agarose gels. The bands were excised and purified using the QIAquick gel extraction kit (Qiagen). Direct sequencing was performed as above.

In addition to CoVs, all 5 samples were tested using PCR assays for other virus groups that are known to induce syncytial cell formation. Primers and protocols for the broad detection of herpesviruses, paramyxoviruses, and reoviruses were used as previously described (Vandevanter et al. 1996, Nollens et al. 2007, Wellehan et al. 2009).

Phylogenetic analysis. The sequences generated using primers $2 \mathrm{Bp}$ and $4 \mathrm{Bm}$ were compared to those in GenBank (National Center for Biotechnology Information), EMBL (Cambridge, UK), and DDBJ (Mishima, Shizuoka, Japan) databases using BLASTN (Altschul et al. 1997). Homologous 208 to 220 nucleotide sequences and predicted amino acid sequences of nidoviral RdRp were aligned using 3 methods: ClustalW2 (Larkin et al. 2007), T-Coffee (Notredame et al. 2000), and MUSCLE (Edgar 2004). Bayesian analyses of the predicted nucleotide alignment were performed using MrBayes 3.1 (Ronquist \& Huelsenbeck 2003) with gamma distributed rate variation and a proportion of invariant sites, 
and a general time reversible model. The first $10 \%$ of 1000000 iterations were discarded as a burn in. Maximum likelihood (ML) analyses of each alignment were performed using PHYLIP (Phylogeny Inference Package, version 3.66) (Felsenstein 1989), running each alignment using the DNAml program with global rearrangements, 5 replications of random input order, and gamma plus invariant rate distributions. The values for the gamma distribution were taken from the Bayesian analysis. Equine torovirus (GenBank accession no. X52374), a member of the family Coronaviridae outside of the genus Coronavirus, was designated as the outgroup. The alignment was used to create data subsets for bootstrap analysis to test the strength of the tree topology (200 re-samplings) (Felsenstein 1985).

\section{RESULTS}

\section{Gross and histological findings}

HS 1, 5, 6, and 17 were adult males; HS 4 and 62 were adult females. Blubber depths ranged from 12 to $20 \mathrm{~cm}$, indicating moderate nutritional status. Each seal had severely congested lungs, with areas of consolidation and hemorrhage and blood-tinged foam in the trachea and bronchi. The mediastinal, tracheobronchial, and submandibular lymph nodes and tonsils were grossly enlarged and hemorrhagic. The gastrointestinal tracts were empty, with traces of feces in the rectum. The histopathological findings in HS D62 included mild multifocal ulcerative gastritis, acute congestion of the cerebellum and brainstem, germinal center depletion and/or histiocytosis with syncytial cell formation in the tonsils, and a peripheral lymph node (Fig. 1). The lung was not submitted for histologic examination. Syncytial cells did not have either intranuclear or intracytoplasmic inclusion bodies. HS 4, 5, and 17 had acute severe necrotizing lymphocytic and histiocytic lobar pneumonia without syncytia, fibrinoid vasculitis, thrombosis, septal edema, and intralesional mixed gram-positive and gram-negative bacteria. In addition, HS 17 had evidence of gram-negative bacterial sepsis. Pseudomonas aeruginosa was cultured from all 3 of these seals (Gaffney et al. 2008). As was the case in D62, germinal centers in lymphoid tissues were often hyalinazed, histiocytic or depleted. Rare syncytia were observed in the lymph nodes of HS 5, but not HS 4 or HS 17.

\section{Degenerate and specific PCR}

PCR amplification using the degenerate primer pair $2 \mathrm{Bp}$ and $4 \mathrm{Bm}$ resulted in a $208 \mathrm{bp}$ product, after editing out of primer sequences, in 1 of the 5 harbor seal lung
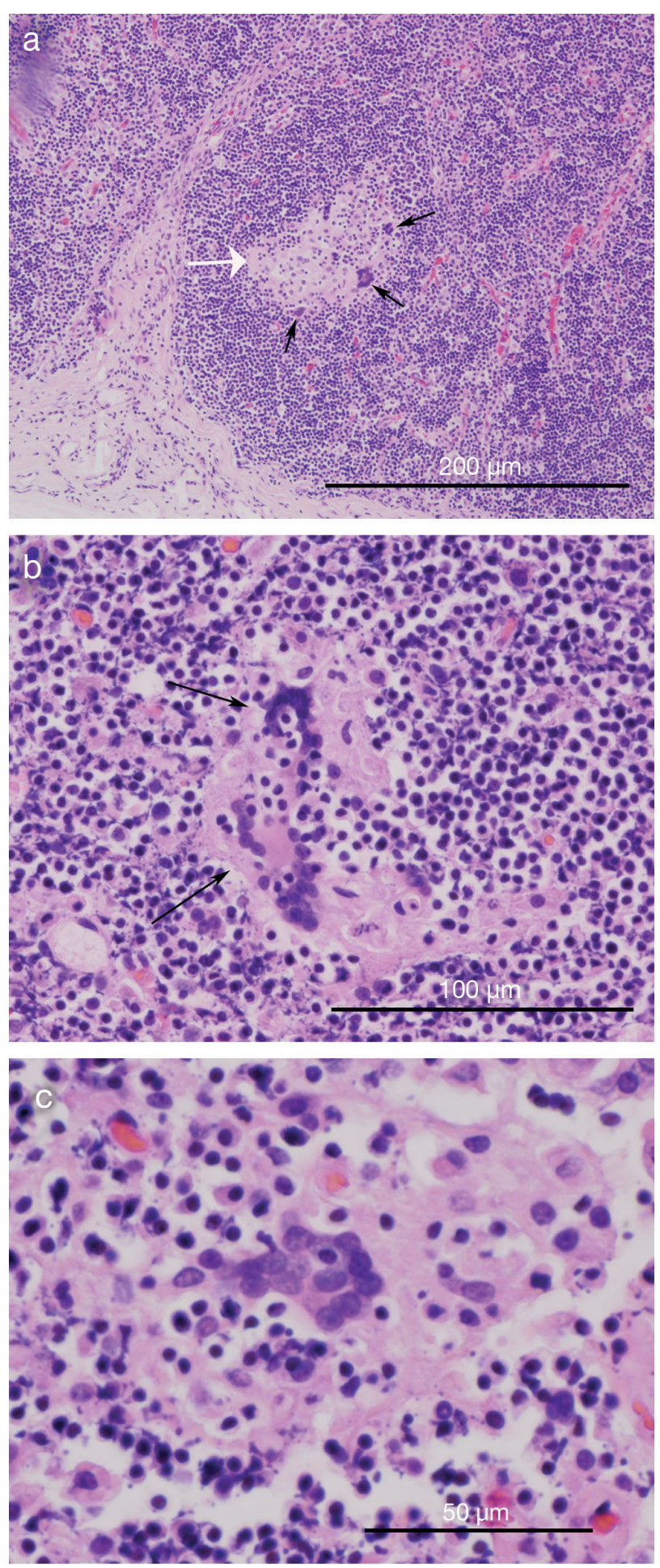

Fig. 1. Phoca vitulina richardsii. (a) Spleen from a harbor seal (HS D62) that died during the 2000 outbreak. Note the germinal center depletion (white arrow) and the presence of multinucleated syncytial cells (black arrows) (H\&E). (b) Tonsil from HS D62. Note the multinucleated syncytial cells (arrows) within a germinal center (H\&E). (c) Tonsil from HS D62. Note the multinucleated syncytial cell in the depleted germinal center of a secondary follicle (H\&E) 
EqToro WhBreamV HSCOV FelCoV FerECoV MuniaCoV TGEV HuCoVNL CanCoV GooseCoV IBV PigeonCoV MuHV BelugaCoV BovCoV BatCoV512 ALC BatCoVHKU6 BatCoVHKU9 BatCoVHKU4 BatCoVHKU5 HumCOVHKU1 SARSCOVAS PEDV

EqToro WhBreamV $\mathrm{HSCOV}$ FelCoV FerECoV MuniaCoV TGEV HuCoVNL CancoV GooseCoV IBV PigeonCoV MuHV BelugaCoV BovCoV BatCoV512 ALC BatCoVHKU6 BatCoVHKU9 BatCoVHKU4 BatCoVHKU5 HumCOVHKU1 SARSCOVAS PEDV

ATTGTCAGCTAAAGCAAGAGCAAGAACAGTTTCCTCATGCTCATTTATAGCATCAACAAT AAGACAAAAGAAAACCCGCGTCCGCACACTAGGAGGTTCATCATTCATCACATCATCAAT CGTCTCTGCCAAGGCTAGGGCTCGCACTGTTGGTGGCGTGTCACTCCTGTCCACAATGAC TATTTCTGGAAAGGCAAGAGCTCGTACTGTCGGAGGAGTGTCGCTTCTCTCAACAATGAC CATATCAGGTAAGGCACGAGCTCGTACTGTTGGTGGTGTGTCACTTTTGTCAACTATGAC GATTTCAGCAAAAGATCGTGCTCGCACTGTTGCAGGTGTTTCTATTATAAGCACCATGAC TATTTCTGGTAAGGCAAGAGCTCGTACAGTAGGAGGAGTTTCACTTCTTTCTACCATGAC TATTAGTGGTAAAGAACGTGCTAGAACTGTTGGTGGTGTTTCTCTGTTGTCCACAATGAC TATTTCTGGAAAGGCTAGAGCTCGTACAGTAGGAGGAGTTTCACTCTTTTCTACTATGAC TATATCTGCAAAGAATAGGGCTCGTACCGTTGCAGGTGTATCTATATTATCTACTATGAC CATATCCGCGAAAAATAGAGCGCGTACAGTGGCAGGTGTGTCTATCCTTTCTACTATGAC AATTTCTGCTAAAAATAGAGCAAGAACTGTTGCTGGTGTTTCAATCCTTTCTACTATGAC TATTAGTGCTAAGAATAGGGCCCGCACCGTTGCTGGTGTCTCTATTCTCAGTACTATGAC CATTTCTGCTAAGTCACGTGCACGTACTGTTGCAGGTGTGTCAATTCTTAGCTCGATGAC TATTAGTGCTAAGAATAGAGCCCGCACTGTTGCTGGTGTTTCCATACTTAGTACTATGAC CATTAGTGGTAAAGATCGAGCACGCACTGTTGGTGGTGTTTCACTCTTGTCTACCATGAC AATTTCAGCCAAGGATCGTGCTCGCACTGTGGCAGGAGTGTCTATAATTAGCACCATGAC TATTAGTGGCAAAGAACGTGCACGTACTGTTGGTGGTGTTTCCTTGCTCTCCACTATGAC TATAAGTGCTAAAAATCGCGCTCGCACTGTAGCTGGTGTTTCTATAGCATCTACTATGAC TATTAGTGCTAAGAATAGAGCACGTACTGTAGCAGGCGTGTCTATACTTAGCACAATGAC TATTAGTGCTAAGAATAGAGCCCGGACTGTTGCCGGTGTGTCGATTCTTAGTACAATGAC TATCAGTGCTAAGAATAGAGCTCGCACTGTAGCAGGTGTTTCTATTCTTAGTACTATGAC CATTAGTGCAAAGAATAGAGCTCGCACCGTAGCTGGTGTCTCTATCTGTAGTACTATGAC TATTAGTGGCAAAGAACGTGCACGCACAGTGGGTGGTGTCTCGCTTTTGTCAACCATGAC

61

ATTTAGATTTGCACATAAACCAGTAACATCTAAAATGGTTGAAGTAGCACAGAATTCAGG CTTCCGCATGCTCCACAAACCAGTCACCAACAAAATGGTCCAAACAGCCCAAGCAAACAT TACCAGACAGTACCATCAAAAGCACCTCAAGTCAATCGCAGCTGCGCGTAATGCTA - - - AACGAGACAGTACCATCAGAAGCATTTAAAATCCATCGCTGCTACACGTAATGCCA - - CACAAGACAGTATCATCAGAAACACTTAAAGCCTATTGCCGCCATGCGTAACGCTA - - - TAACAGGCAGTACCATCAGAAGATTCTGAAATCGATTTCACTTGCACGCAATCAGA - - - TACGAGACAATATCATCAGAAGCATTTGAAGTCAATTGCTGCAACACGCAATGCTA- - - CACAAGACAATACCATCAAAAACATCTTAAATCCATTGTTAATACACGCAATGCCA - - TACGAGACAATACCACCAGAAGCATTTAAAGTCAATTGCTGCAACACGCAATGCCA - - TAATAGACAGTATCACCAGAAGGTGCTTAAGTCTATTGTTAACACACGTAATGCAG - - TAATAGGCAGTTTCATCAGAAGATTCTTAAGTCTATAGTCAACACTAGAAACGCTC - - CAATAGGCAATATCATCAAAAGGTGCTTAAGTCTATTGTTAATACTAGAAATGCTC - - - TGGCAGAATGTTTCATCAAAAGTGTCTAAAGAGTATAGCAGCTACTCGCGGTGTTC - - - AAACCGCCAATTTCATCAGAAGTGCCTCAAATCTATTGTGAACACGCGGAATGCAA - - TGGCAGAATGTTTCATCAAAAATGTTTGAAAAGTATAGCAGCTACACGTGGTGTTC - - - TACTAGACAATACCACCAGAAACACCTGAAATCTATTGTCAACACTAGAGGTGCTT - - - TAACAGGCAGTACCATCAAAAGATGCTGAAATCTATTTCACTTGCACGCAATCAGA - - - CACTAGACAGTATCACCAGAAGCATTTGAAGTCTATTGTCAACACGCGCGGTGCGT - - - AAATAGACAATTTCATCAGAAGATGCTTAAGTCTATAGCTGCAGCACGTGGTGCAT - - - TAATCGTCAATATCACCAGAAGATGTTGAAGTCTATGGCTGCAACGCGTGGAGCCA - - TAATCGTCAATACCACCAGAAAATGCTTAAGTCTATGGCTGCTACGCGGGGTTCCA - - AGGCCGAATGTTCCATCAAAAATGTTTGAAGAGTATAGCAGCTACCCGAGGTGTTC - - - AAATAGACAGTTTCATCAGAAATTATTGAAGTCAATAGCCGCCACTAGAGGAGCTA - - - CACTCGGCAGTATCATCAGAAACACCTTAAATCCATAGTTAATACTAGGGGCGCTT - - - -

Fig. 2. Nucleotide alignment of the partial harbor seal coronavirus (HSCoV; 208 bp; in bold) and homologous coronavirus RNAdependent RNA polymerase (RdRp) sequences. Conserved nucleotides are indicated. Sequences retrieved from GenBank include Equine torovirus (EqToro; DQ310701), White bream virus (WhBreamV; DQ898157), Feline coronavirus (FelCoV; GenBank accession no. DQ848678), Ferret enteric coronavirus (FerECoV; DQ340560), Munia coronavirus (MuniaCoV; FJ376622), Transmissible gastroenteritis virus (TGEV; DQ811789), Human coronavirus NL (HuCoVNL; AY518894), Canine coronavirus (CanCoV; EU856362), Goose coronavirus (GooseCoV; AJ854123), Infectious bronchitis virus (IBV; AY851295), Pigeon coronavirus (Pigeon$\left.\mathrm{CoV}_{;} \mathrm{AY} 851295\right)$, Murine hepatitis virus (MuHV; AF208067), Beluga whale coronavirus (BelugaCoV; NC_010646), Bovine coronavirus (BovCoV; DQ811784), Scotophilus bat coronavirus 512 (BatCoV512; DQ648858), Asian leopard cat coronavirus (ALC; EF584908), Myotis bat coronavirus HKU6 (BatCoVHKU6; DQ249224), Rousettus bat coronavirus HKU9 (BatCoVHKU9; EF065513), Tylonycteris bat coronavirus HKU4 (BatCoVHKU4; DQ249214), Pipistrellus bat coronavirus HKU5 (BatCoVHKU5; DQ249217), Human coronavirus HKU1 (HumCoVHKU1; AY597011), SARS coronavirus (SARSCoVAS; AY427439), Porcine epidemic diarrhea virus (PEDV; AF353511) 


\begin{tabular}{|c|c|}
\hline & $\begin{array}{ll}121 & 180\end{array}$ \\
\hline EqToro & TGGCTTTTGCCTCATTGGTGTATCCAAGTATGGTTTAAAGTTTTCAAAGTTTTTGAAAGA \\
\hline WhBreamV & CGGCCCCTTTCTAATTGGAATTTCAAAATTCAACCTCGGATTTCACAAATACCTCTCCGC \\
\hline HSCOV & - - - - CAGTAGTCATAGGGACCACAAAGTTCTATGGCGGCTGGGAYGCCATGTTGAAAAA \\
\hline Fel CoV & - - - - CTGTTGTTATAGGAACTACCAAGTTCTATGGTGGCTGGGATAACATGTTAAAGAA \\
\hline FerECoV & - - - - CAGTTGTCATTGGTACAGCCAAGTTTTACGGCGGATGGGACGATATGTTAAAGAA \\
\hline MuniaCoV & - - - - СTATAGTAATAGGCACTACCAAGTTTTATGGTGGTTGGGATAATATGTTACGTAG \\
\hline TGEV & - - - - CTGTGGTCATTGGTTCAACCAAGTTTTATGGTGGTTGGGACAATATGCTTAAAAA \\
\hline HuCOVNL & - - - - CTGTTGTTATTGGTACTACCAAATTTTATGGTGGTTGGAATAATATGTTGCGTAC \\
\hline CanCoV & - - - - CTGTGGTCATTGGTTCAACCAAGTTTTATGGTGGTTGGGATAACATGCTTAAAAA \\
\hline GooseCoV & - - - - СTGTTGTTATCGGTACATCCAAGTTCTATGGAGGCTGGGACAACATGTTGCGCA \\
\hline IBV & - - - - CTGTAGTTATTGGAACAACCAAGTTTTATGGCGGTTGGGATAACATGTTGAGAA \\
\hline PigeonCoV & - - - - СTGTTGTTATAGGAACTACCAAGTTTTATGGTGGTTGGGATAACATGTTAAGAAA \\
\hline MuHV & - - - - CTGTAGTTATAGGCACCACGAAGTTCTATGGCGGTTGGGATGATATGTTACGCCG \\
\hline BelugaCov & - - - - CCGTTGTAATAGGCACTACTAAGTTTTATGGAGGATGGGATAATATGCTCCGCAA \\
\hline BovCoV & - - - - СTGTTGTTATAGGCACCACTAAGTTTTATGGCGGCTGGGATGATATGTTACGTCG \\
\hline BatCoV512 & - - - - CTGTTGTTATTGGAACCACTAAGTTCTATGGTGGTTGGGATAATATGCTCAAAAC \\
\hline ALC & - - - CCATCGTGATTGGAACAACCAAATTCTATGGTGGTTGGGACAACATGTTACGACG \\
\hline BatCoVHKU6 & - - - - CTGTTGTTATAGGTACTACAAAATTTTATGGTGGCTGGGATAATATGCTTAAGAC \\
\hline BatCoVHKU9 & - - - - СTGTTGTTATAGGTACCACTAAATTTTATGGAGGGTGGAATCGCATGCTTCGCAC \\
\hline BatCoVHKU4 & - - - - CGTGTGTCATAGGCACAACTAAATTTTATGGTGGTTGGGACTTTATGTTAAAAAC \\
\hline BatCoVHKU5 & - - - - CGTGCGTCATAGGGACCACTAAGTTCTATGGTGGTTGGGACTTTATGTTAAAAAC \\
\hline HumCOVHKU1 & - - - - СTGTTGTTATAGGAACCACTAAATTTTATGGTGGTTGGGACGATATGTTACGTC \\
\hline SARSCOVAS & - - - - CTGTGGTAATTGGAACAAGCAAGTTTTACGGTGGCTGGCATAATATGTTAAAAAC \\
\hline \multirow[t]{2}{*}{ PEDV } & - - - - CGGTTGTTATTGGTACTACTAAGTTTTATGGTGGTTGGGACAATATGCTTAAGAA \\
\hline & $181 \quad 220$ \\
\hline EqToro & TAAGTAC- - -GGTGCTATTGAGGGTTTTGATGTGTTTGGT \\
\hline WhBreamV & TCACCATCCAAACGGAATAGAAGACTGTCAAGTTATGGGT \\
\hline HSCOV & TCTCACT-- -TTAGGCGTAGATAATGGTTGCCTTATGGGT \\
\hline FelCoV & TTTAATG - - CGTGATGTAGACAATGGTTGTTTGATGGGA \\
\hline FerECoV & TTTGATG - - CGTGACGTTGATAATGGCTGTCTTATGGGT \\
\hline MuniaCoV & GCTGATG- - - CACAATATCAACAATCCCATATTAGTGGGC \\
\hline TGEV & TTTAATG- - - CGTGATGTTGATAATGGTTGTTTGATGGGA \\
\hline HuCOVNL & TTTAATT- - -GATGGTGTTGAAAACCCTATGCTCATGGGT \\
\hline CanCoV & TTTAATG - - CGTGACGTTGATAATGGTTGTTTGATGGGA \\
\hline GooseCoV & TCTTATT- - -GGTGGCGTTGATAATCCTATGCTTATGGGC \\
\hline IBV & ССTTATT - - CAGGGTGTTGAAGACCCGATTCTTATGGGT \\
\hline PigeonCoV & ССТCATT- - -AAAGGTGTTGATGACCCCATTCTTATGGGA \\
\hline MuHV & ССTTATT- - -AAAGATGTTGATAGTCCTGTACTCATGGGT \\
\hline BelugaCoV & ССTTATG- - - CGCGGTGTGGAGGATCCTGTTCTGATGGGG \\
\hline BovCoV & ССТTATT - - АAAGATGTTGATAATCCTGTACTTATGGGT \\
\hline BatCoV512 & АСТTATT- - -AAGGATGTTGAAAACCCCCATTTAATGGGA \\
\hline $\mathrm{ALC}$ & ACTGATG - - TGTAATATCAACAATCCCATTTTAGTGGGT \\
\hline BatCoVHKU6 & АСTTATT - - GGTGATGTGGATAACCCGAATCTTATGGGT \\
\hline BatCoVHKU9 & TTTGTGT - - -GAAGGTGTAGAAAATCCACATTTGATGGGT \\
\hline BatCoVHKU4 & СТTATAT - - AAGGATGTTGAGAGTCCACATTTAATGGGT \\
\hline BatCoVHKU5 & CTTGTAT - - AAGGATGTAGATAATCCTCACTTGATGGGT \\
\hline HumCOVHKU1 & TCTTATA---AAGGATGTTGACAACCCTGTTCTTATGGGT \\
\hline SARSCOVAS & TGTTTAC- - -AGTGATGTAGAAACTCCACACCTTATGGGT \\
\hline PEDV & CСTTATT- - -GATGGTGTTGAAAATCCGTGTCTTATGGGT \\
\hline
\end{tabular}

Fig. 2 (continued)

samples (HS 1). Specific PCR on the same sample using the specific primers HSCoV-F1 and HSCoV-R1 yielded an amplicon of $148 \mathrm{bp}$, after editing out of primers. The 208 bp sequence was submitted to GenBank under accession no. FJ766501. No coronaviral RNA was amplified from the other 4 harbor seals (HS 4, 5,6 , and 17) with either the degenerate or the specific PCR. No paramyxoviral or reoviral RNA was detected in any of the samples.

\section{Phylogenetic analysis}

BLASTN results showed the highest score with Feline coronavirus (GenBank accession no. DQ848678). Results of all 3 alignment methods were identical, with no indels present when either nucleotide or predicted amino acid Coronavirus sequences were aligned. When additional nidoviruses equine torovirus and white bream virus (GenBank accession no. DQ898157) 
were added, predicted amino acid alignments were identical by all 3 methods, and the nucleotide alignment based on the predicted amino acid alignment was used for analysis (Fig. 2). The Bayesian tree is shown in Fig. 3. Bootstrap values from ML analysis are shown on the tree.

\section{DISCUSSION}

Here we report on the identification of a novel respiratory $\mathrm{CoV}$ from a Pacific harbor seal. Our phylogenetic analysis places this virus as a basally divergent Group 1a CoV. It is distinct from, but most closely related to, the CoVs of ferrets, cats, dogs, and swine. While inclusion of this virus in Group 1a would create greater diversity within this one subgroup than is seen in any other subgroup, this clustering of $\mathrm{HSCoV}$ with the CoV of ferrets, cats, dogs, and swine is supported. There does not appear to be a clear demarcation for separating it into distinct subgroups.

Because this is a retrospective investigation and due to the incomplete sampling at the time of the outbreak, the clinical significance of $\mathrm{HSCoV}$ cannot fully be determined. However, the involvement of $\mathrm{HSCoV}$ in the etiology of the epizootic pneumonia and resulting die-off of the harbor seals is suspected. Several CoV have a known tropism for the lower respiratory tract, and porcine, bovine, canine, and human respiratory CoVs have demonstrated the ability to cause epizootics and the potential to cause fatalities (Erles et al. 2003, Costantini et al. 2004, Decaro et al. 2008). The reported histopathological changes, including the formation of syncytia or giant cells, is consistent with those associated with other $\mathrm{CoV}$, such as porcine respiratory coronavirus and SARS virus (Kusanagi et al. 1992, Franks et al. 2003). However, HSCoV was only detected in 1 of 5 analyzed lung samples, and no samples were collected from HS D62 for molecular analysis. The intralesional bacteria and Pseudomonas sp. likely represent opportunistic overgrowth and secondary invaders, suggesting that the lung samples were not collected during the acute phase of the infection, although this cannot be confirmed from the samples available. The window of detection of $\mathrm{CoV}$ in respiratory infections can be short. Only 1 in 3 piglets sheds detectable porcine respiratory $\mathrm{CoV}$ levels by $5 \mathrm{~d}$ following first clinical signs (Costantini et al. 2004). Similarly, shedding of the human SARS virus peaks 7 to $10 \mathrm{~d}$ after onset of clinical signs (Poon et al. 2004). It is possible that $4 \mathrm{HSCoV}$ negative harbor seals had cleared the initial HSCoV infection or that the virus load in those lung samples had tapered beyond the detection limit of both our degenerate and specific PCR assay. A similar event had occurred 3 yr earlier, in 1997, when approximately 90 seals were found dead in the same area.

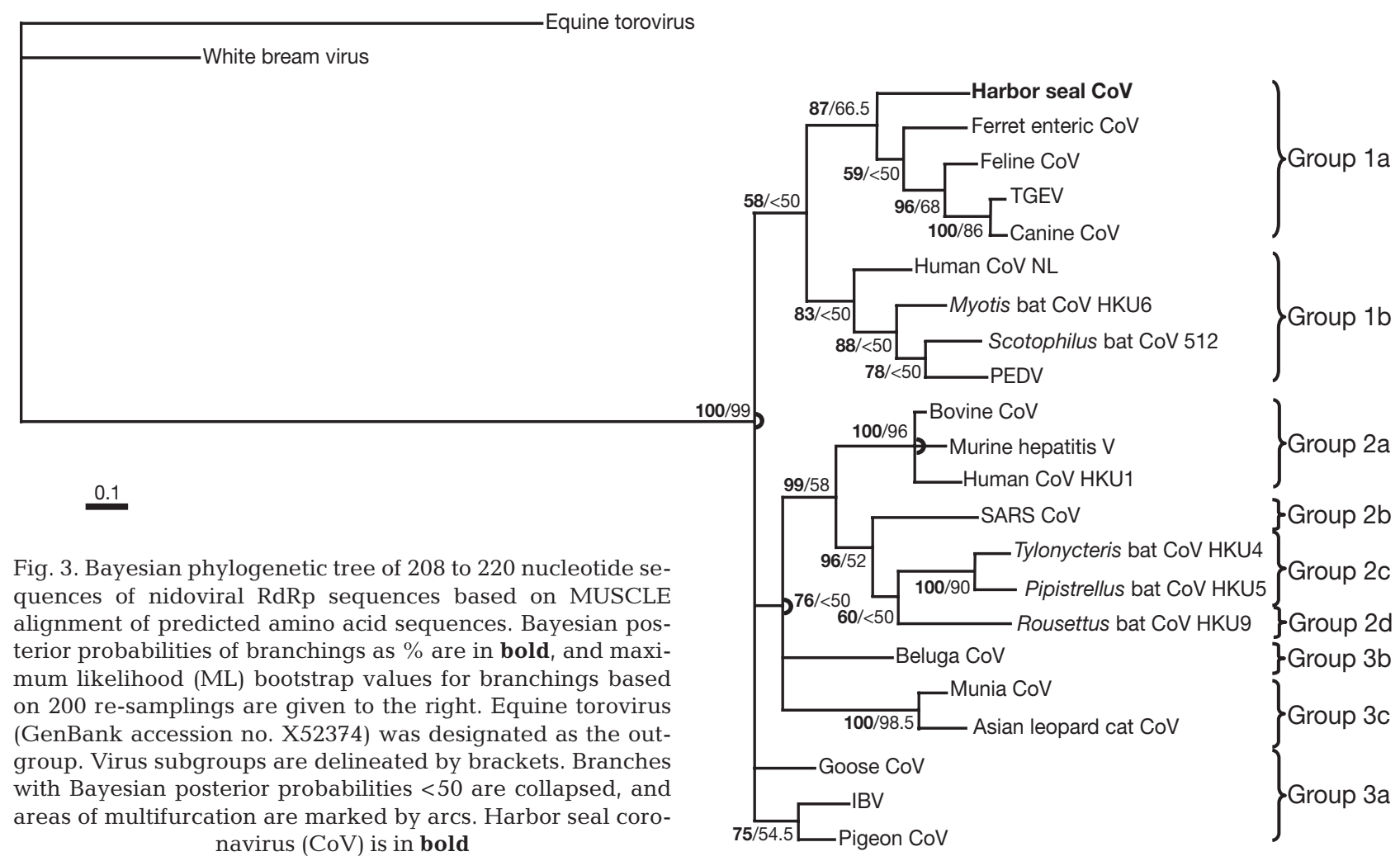


Necropsy and histopathological analysis of 7 of these seals yielded similar gross and histopathological presentations (authors' unpubl. data) to the 5 cases included in this study. During this earlier epizootic, syncytia formation was observed in the lungs of 3 animals and in the adrenal, liver, and salivary gland of 2 other animals. The causative agent for the 1997 respiratory infections and fatalities was never determined. The involvement of HSCoV in the 1997 epizootic is a distinct possibility. However, no suitable samples are available for retrospective $\mathrm{HSCoV}$ testing from this event. Thus, archiving of tissues from wildlife involved in die-offs is highly recommended, so later retrospective investigations using more modern techniques can be employed.

We were able to generate only limited genomic HSCoV sequence (208 bp), and the exact phylogenetic placement of $\mathrm{HSCoV}$ is therefore provisional. Attempts to amplify flanking sequences using combinations of specific and degenerate primers were unsuccessful. However, the overall topology and branching order of the phylogenetic tree (Fig. 3) based on the $208 \mathrm{bp}$ $\mathrm{HSCoV}$ and $\mathrm{CoV}$ homologous sequences are consistent with the generally accepted phylogenetic branching order of the CoVs (Decaro \& Buonavoglia 2008, Woo et al. 2009). We do note that our phylogenetic analysis does not support the monophyly of the proposed Subgroups $3 \mathrm{~b}$ and $3 \mathrm{c}$ with $3 \mathrm{a}$, and finds weak support of monophyly of these subgroups with Group 2. Previous analyses have found that Subgroups 3b and 3c were most closely related to the Group 3a viruses (Mihindukulasuriya et al. 2008, Woo et al. 2009). These differences may be due to the inclusion of non-Coronavirus nidoviral outgroup rooting. If the ancestral coronavirus node were within the proposed Group 3, then lack of an appropriate outgroup for rooting may cause basal viruses to appear monophyletic. Other analyses that have used non-Coronavirus nidoviral outgroup rooting have found that Group 3a CoVs appear fairly basal (Schütze et al. 2006, Wise et al. 2006), and this would make sense if there was an early divergence between viruses using mammalian hosts in Groups 1 and 2 and those using avian hosts in Group 3. However, these other analyses did not include viruses in the proposed Subgroups $3 \mathrm{~b}$ and 3c. The major limitation of this approach is that there are limited regions that can be reliably aligned with non-Coronavirus nidoviruses. Examination of these further regions and additional taxa may confirm or refute monophyly of these subgroups.

Acknowledgements. The authors thank the staff and volunteers of the Marine Mammal Center, especially Denise Greig. All sample collection protocols were approved by the University of Florida Institutional Animal Care and Use Committee (IACUC\#C233).

\section{LITERATURE CITED}

Altschul SF, Madden TL, Schäffer AA, Zhang J, Zhang Z, Miller W, Lipman DJ (1997) Gapped BLAST and PSIBLAST: a new generation of protein database search programs. Nucleic Acids Res 25:3389-3402

Carrington CV, Foster JE, Zhu HC, Zhang JX and others (2008) Detection and phylogenetic analysis of group 1 coronaviruses in South American bats. Emerg Infect Dis $14: 1890-1893$

> Costantini V, Lewis P, Alsop J, Templeton C, Saif LJ (2004) Respiratory and fecal shedding of porcine respiratory coronavirus (PCRV) in sentinel weaned pigs and sequence of the partial S-gene of the PCRV isolates. Arch Virol 149:957-974

$>$ Decaro N, Buonavoglia C (2008) An update on canine coronaviruses: viral evolution and pathobiology. Vet Microbiol 132:221-234

Decaro N, Mari V, Desario C, Campolo M and others (2008) Severe outbreak of bovine coronavirus infection in dairy cattle during the warmer season. Vet Microbiol 126:30-39

Edgar RC (2004) MUSCLE: multiple sequence alignment with high accuracy and high throughput. Nucleic Acids Res 32:1792-1797

$>$ Erles K, Toomey C, Brooks HW, Brownlie J (2003) Detection of a group 2 coronavirus in dogs with canine infectious respiratory disease. Virology 310:216-223

Felsenstein J (1985) Confidence limits on phylogenies: an approach using the bootstrap. Evolution 39:783-791

Felsenstein J (1989) PHYLIP-Phylogeny inference package. Cladistics 5:164-166

Franks TJ, Chong PY, Chui P, Galvin JR and others (2003) Lung pathology of severe acute respiratory syndrome (SARS): a study of 8 autopsy cases from Singapore. Hum Pathol 34:743-748

Gaffney PM, Colegrove KM, Gulland FMD, Byrne B, Jang SS, Edgar K, Lowenstine LJ (2008) Pathologic, microbiologic and epidemiologic characterization of Pseudomonas sp. in California sea lions (Zalophus californianus) and Pacific harbor seals (Phoca vitulina). In: Reidarson TH (ed) Proceedings of the 39th Annual Conference of the International Association for Aquatic Animal Medicine, May 10-14, 2008, Rome. IAAAM, Rome, p 100

González JM, Gomez-Puertas P, Cavanagh D, Gorbalenya AE, Enjuanes L (2003) A comparative sequence analysis to revise the current taxonomy of the family Coronaviridae. Arch Virol 148:2207-2235

> Kusanagi K, Kuwahara H, Katoh T, Nunoya T, Ishikawa Y, Samejima T, Tajima M (1992) Isolation and serial propagation of porcine epidemic diarrhea virus in cell cultures and partial characterization of the isolate. J Vet Med Sci 54: 313-318

Larkin MA, Blackshields G, Brown NP, Chenna R and others (2007) ClustalW and ClustalX version 2. Bioinformatics 23:2947-2948

Li W, Shi Z, Yu M, Ren W and others (2005) Bats are natural reservoirs of SARS-like coronaviruses. Science 310: $676-679$

Mihindukulasuriya KA, Wu G, St Leger J, Nordhausen RW, Wang D (2008) Identification of a novel coronavirus from a beluga whale by using a panviral microarray. J Virol 82:5084-5088

Nollens HH, Wellehan JX, Saliki JT, Caseltine SL, Jensen ED, Van Bonn W, Venn-Watson S (2007) Characterization of a parainfluenza virus isolated from a bottlenose dolphin (Tursiops truncatus). Vet Microbiol 128:231-242

Notredame C, Higgins DG, Heringa J (2000) T-Coffee: a 
novel method for fast and accurate multiple sequence alignment. J Mol Biol 302:205-217

Poon LLM, Chan KH, Wong OK, Cheung TKW and others (2004) Detection of SARS coronavirus in patients with severe acute respiratory syndrome by conventional and real-time quantitative reverse transcription-PCR assays. Clin Chem 50:67-72

Ronquist F, Huelsenbeck JP (2003) MrBayes 3: Bayesian phylogenetic inference under mixed models. Bioinformatics 19:1572-1574

Saif LJ (2004) Animal coronaviruses: What can they teach us about the severe acute respiratory syndrome? Rev Sci Tech 23:643-660

Schütze H, Ulferts R, Schelle B, Bayer S and others (2006) Characterization of white bream virus reveals a novel genetic cluster of nidoviruses. J Virol 80:11598-11609

Stephensen CB, Casebolt DB, Gangopadhyay NN (1999) Phylogenetic analysis of a highly conserved region of the polymerase gene from 11 coronaviruses and development of a consensus polymerase chain reaction assay. Virus Res

Editorial responsibility: Michael Moore, Woods Hole, Massachusetts, USA
60:181-189

> Vandevanter DR, Warrener P, Bennett L, Schultz ER, Coulter S, Garber RL, Rose TM (1996) Detection and analysis of diverse herpesviral species by consensus primer PCR. J Clin Microbiol 34:1666-1671

- Wellehan JF Jr., Childress AL, Marschang RE, Johnson AJ and others (2009) Consensus nested PCR amplification and sequencing of diverse reptilian, avian, and mammalian orthoreoviruses. Vet Microbiol 133:34-42

Wise AG, Kiupel M, Maes RK (2006) Molecular characterization of a novel coronavirus associated with epizootic catarrhal enteritis (ECE) in ferrets. Virology 349:164-174

> Woo PC, Wang M, Lau SK, Xu H and others (2007) Comparative analysis of twelve genomes of three novel group 2c and group $2 \mathrm{~d}$ coronaviruses reveals unique group and subgroup features. J Virol 81:1574-1585

Woo PC, Lau SK, Lam CS, Lai KK and others (2009) Comparative analysis of complete genome sequences of three avian coronaviruses reveals a novel group 3c coronavirus. J Virol 83:908-917

Submitted: February 23, 2009; Accepted: December 14, 2009 Proofs received from author(s): May 24, 2010 